\title{
WI - Call for Papers Heft 3/2013
}

\section{Wirtschaftsinformatik und Marketing}

DOI 10.1007/s11576-011-0302-2

\section{Die Autoren}

Prof. Dr. Martin Spann ( $\varangle)$

Institut für Electronic Commerce und Digitale Märkte

Ludwig-Maximilians-Universität

München

80539 München

Deutschland

spann@bwl.Imu.de

Prof. Dr. Oliver Hinz

Professur für Wirtschaftsinformatik -

Electronic Markets

Technische Universität Darmstadt

64289 Darmstadt

Deutschland

hinz@wi.tu-darmstadt.de

Prof. Dr. Vandana Ramachandran

Operations and Information Systems

David Eccles School of Business

University of Utah

SLC 84112 Utah

USA

vandana@business.utah.edu

Online publiziert: 2011-11-03

This article is also available in English via http://www.springerlink.com and http://www.bise-journal.org: Spann M, Hinz O, Ramachandran V (2011) BISE - Call for Papers, Issue 3/2013. BISE and Marketing. Bus Inf Syst Eng. doi: 10.1007/s12599-011-0193-1.

(c) Gabler Verlag 2011

\section{Schwerpunktthema}

Elektronische Medien haben zu völlig neuen Möglichkeiten und Herausforderungen für das Marketing geführt. Im Vergleich zu traditionellen Medien ermöglichen digitale Medien eine wesentlich bessere Messung der Effektivität und
Effizienz von Werbung. Darüber hinaus fallen umfangreiche Daten des Nutzerverhaltens an, die zur Personalisierung von Produkten, Werbung und der individuellen Betreuung von Kundenbeziehungen ebenso genutzt werden können wie die direkte Interaktion mit Kunden über neuartige Medien. Es sind neue Möglichkeiten zur Vermarktung von Produkten im Rahmen von Suchmaschinen, sozialen Netzwerken und mobilen Endgeräten entstanden. Die Interaktivität elektronischer Medien hat weiterhin zu einer Verbreitung interaktiver Preismechanismen wie beispielsweise Auktionen geführt. Diese Innovationen wurden durch die Entwicklungen im Bereich der Informationssysteme ausgelöst. Deshalb bietet insbesondere die Schnittstelle zwischen Wirtschaftsinformatik und Marketing großes Potenzial für die Forschung. Das Ziel dieses Schwerpunktthemas ist daher, Forschung an dieser Schnittstelle voran zu bringen.

Beiträge aus Forschung und Praxis sind u. a. zu folgenden (gerne auch weiteren) Themenfeldern erwünscht:

- Customer Relationship Management

- Suchmaschinenmarketing

- Marketing mit mobilen Endgeräten

- Online Pricing

- Auktionen

- Marketing in sozialen Netzwerken

- User Generated Content

- Gestaltung von Marketing-bezogenen Informationssystemen

- Suchmaschinenoptimierung

- Entscheidungsunterstützungssysteme im Marketing

- Design Science in Electronic Commerce

- Open Innovation und Crowd Sourcing

- Interaktion zwischen IT- und Marketingabteilungen

\section{Einreichung von Beiträgen}

Bitte reichen Sie Beiträge für die Rubriken WI - Aufsatz und WI - State of the Art bis spätestens 2012-07-01 über das Online-Begutachtungssystem (http://www.editorialmanager.com/buis/) ein. Bitte beachten Sie die Hinweise $\mathrm{zu}$ formaler Gestaltung und Umfang von Beiträgen für die WIRTSCHAFTSINFORMATIK/Business \& Information Systems Engineering (BISE). Vollständige Beiträge sollten höchstens 50.000 Zeichen einschließlich Leerzeichen umfassen, abzüglich 5.000 Zeichen je Seite an Bildern. Ausführliche Autorenrichtlinien stehen unter http://www.wirtschaftsinformatik.de zum Download bereit.

Eingereichte Beiträge werden anonymisiert von mehreren Gutachtern in einem doppelt-blinden Verfahren auf Relevanz, Originalität und fachliche Qualität beurteilt. Neben den Herausgebern des Schwerpunktthemas und jenen der Zeitschrift wirken dabei weitere ausgewiesene internationale Persönlichkeiten aus Wissenschaft und Praxis mit.

Ergänzend zu den Aufsätzen sind auch weitere Einreichungen zum Schwerpunktthema willkommen.

Angenommene Beiträge erscheinen identisch in Deutsch und Englisch. Die deutschsprachige Fassung erscheint in WIRTSCHAFTSINFORMATIK, die englischsprachige in Business \& Information Systems Engineering (BISE).

\section{Zeitplan}

Einreichung von Beiträgen: 2012-07-01 Benachrichtigung der Autoren:

2012-08-26

Abschluss der ersten Überarbeitung:

1012-10-28

Benachrichtigung der Autoren:

2012-12-16

Ggf. Abschluss einer zweiten Überarbeitung (einsprachig): 2013-01-20

Ggf. Abschluss einer zweiten Überarbeitung (zweisprachig): 2013-02-17 Geplanter Erscheinungstermin Heft 3/2013: Juni 2013 